in vivo $32: 1169-1174(2018)$

doi:10.21873/invivo.11360

\title{
Early-stage Clinical Characterization of Malignant Pleural Mesothelioma
}

\author{
YOSHIKI NEGI $^{1 *}$, KOZO KURIBAYASHI ${ }^{1,2^{*}}$, NORIHIKO FUNAGUCHI ${ }^{3}$, HIROSHI DOI $^{4}$, KOJI MIKAMI $^{1}$, \\ TOSHIYUKI MINAMI ${ }^{1}$, TERUHISA TAKUWA ${ }^{5}$, TAKASHI YOKOI ${ }^{2}$, SEIKI HASEGAWA $^{5}$ and TAKASHI KIJIMA ${ }^{1,2}$ \\ ${ }^{1}$ Division of Respiratory Medicine, Department of Internal Medicine, \\ Hyogo College of Medicine, Hyogo, Japan; \\ ${ }^{2}$ Department of Thoracic Oncology, Hyogo College of Medicine, Hyogo, Japan; \\ ${ }^{3}$ Department of Respiratory Medicine, Murakami Memorial Hospital, Asahi University, Hyogo, Japan; \\ ${ }^{4}$ Department of Radiology, Hyogo College of Medicine, Hyogo, Japan; \\ ${ }^{5}$ Department of Thoracic Surgery, Hyogo College of Medicine, Hyogo, Japan
}

\begin{abstract}
Background/Aim: A strategy for improving survival of malignant pleural mesothelioma (MPM) patients is earlier diagnosis paired with earlier stage implementation of therapeutic interventions. This study aimed to determine the clinical signs of early-stage MPM to aid an earlier diagnosis and earlier-stage intervention. Materials and Methods: Out of the 72 cases in our institution, 40 cases with ${ }^{18}$ F-FDG-PET/CT-negative MPM were retrospectively identified between 2007 and 2015. Overall survival rates were determined and compared with pathological features, histology, and treatment. Results: The biphasic histological type of early-stage MPM was characterized by poor prognosis $(p=0.0006)$. Additionally, the cytology-negative group (Class III and below) showed significantly shorter survival times $(p=0.0290)$. There was no significant difference in survival between patients who received pleurectomy and those who received chemotherapy only $(p=0.6991)$. Bimodal therapy resulted in a longer survival rate than trimodal therapy. Conclusion: In early-stage PET-negative MPM cases,
\end{abstract}

This article is freely accessible online.

*These Authors made an equal contribution to the study.

Correspondence to: Dr. Kozo Kuribayashi, Division of Respiratory Medicine, Department of Internal Medicine, Hyogo College of Medicine, 1-1 Mukogawa-cho, Nishinomiya, Hyogo 663-8501, Japan. Tel: +81 798456596, Fax: +81 798456597, e-mail: kuririn@hyo-med.ac.jp

Key Words: Malignant pleural mesothelioma, ${ }^{18} \mathrm{~F}$-fluorodeoxyglucose positron emission computed tomography $\left({ }^{18} \mathrm{~F}-\mathrm{FDG}-\mathrm{PET} / \mathrm{CT}\right)$, early clinical stage, extrapleural pneumonectomy (EPP), pleurectomy or decortication (P/D), biphasic. biphasic histology and pleural effusion of Class III and below correlated with a poor prognosis. Surgical treatment using pleurectomy/decortication resulted in higher patient survival outcomes than therapy with extrapleural pneumonectomy.

The 5-year survival rate of cases undergoing surgery for primary lung cancer in Japan has improved from $51.9 \%$ in 1996 to $69.6 \%$ in 2004 (1). Although this could be attributed to several reasons, early diagnosis and early treatment are regarded as particularly important factors. In fact, $48.1 \%$ of the 11,663 cases that underwent surgery in 2004 in the Lung Cancer Registry Study were in stage Ia, with a 5-year survival rate of $85.1 \%$ (1).

However, malignant pleural mesothelioma (MPM) remains one of the most difficult malignancies to treat, with a median survival time (MST) from diagnosis of about 1 year (2). Treatments shown to lower the mortality or morbidity of MPM include adjuvant chemotherapy (2), neoadjuvant chemotherapy (3), pemetrexed induction therapy $(4,5)$, extrapleural pneumonectomy (EPP), introduction of radical pleural resection/cortical ablation (P/D) (6), adjuvant radiation therapy (7), and introduction of intensity-modulated radiation therapy (IMRT) $(8,9)$. Trimodality therapy (TMT), involving induction chemotherapy followed by EPP and postoperative radiation therapy is currently regarded as the most powerful treatment option (10). However, in most large-scale studies, the MST after TMT still does not reach 20 months $(11,12)$, suggesting that aggressive surgery for MPM patients may not be the most effective option (13). Another strategy for improving survival is earlier diagnosis of MPM paired with earlier stage implementation of therapeutic interventions. Therefore, 40 retrospectively diagnosed MPM cases were investigated to determine the clinical signs of early-stage MPM. 


\section{Materials and Methods}

Study approval. This study was approved by the institutional review board of the Hyogo College of Medicine, Japan in accordance with the Declaration of Helsinki. The requirement for informed consent was waived.

Patients. Seventy-two cases diagnosed as MPM (confirmed by pathology) between April 2007 and December 2015 at the Hyogo College of Medicine in Japan were included. For TNM classification, the International Mesothelioma Interest Group (IMIG) staging was used. The cases in this study were in the early stages, and all cases were N0, M0. Patients were first stratified into a positron emission tomography (PET)-positive group (32 cases) exhibiting significant fluorodeoxyglucose (FDG) accumulation (SUV $\geq 2.5$ ) in FDG-PET at diagnosis, and a PET-negative group (40 cases) not exhibiting significant FDG accumulation. The PETnegative group served as early-stage MPM cases. Patient characteristics (age, sex, status of exposure to asbestos), presence of pleural effusion, cytodiagnosis, histological type, and treatment modalities were analysed.

Integrated ${ }^{18} F-F D G-P E T / C T$. The imaging procedure used in this study has been previously described (14). All ${ }^{18} \mathrm{~F}-\mathrm{FDG}$ PET/computed tomography (CT) scans were performed using a PET scanner combined with a 16-multidetector $\mathrm{CT}$ and gadolinium oxyorthosilicate detectors (Gemini GXL 16; Philips Medical Systems, Eindhoven, The Netherlands). Patients were administered with $4.0 \mathrm{MBq} / \mathrm{kg}$ of body weight of ${ }^{18} \mathrm{~F}-\mathrm{FDG}$ after fasting for $5 \mathrm{~h}$. Static emission images were obtained approximately $60 \mathrm{~min}$ after injection. Helical whole-body CT scans were obtained to attenuate the correction and anatomic localization. Following CT scan completion, PET images from the head to the mid-thigh and the mid-thigh to the tips of the toes were acquired at the $90 \mathrm{~s} / \mathrm{bed}$ position and $30 \mathrm{~s} /$ bed position, respectively, employing a variable sampling method.

Quantitative analysis of FDG uptake was determined by marking a three-dimensional region of interest (ROI) over the most intense area of FDG accumulation in the primary maxillary sinus tumour. The SUV was calculated as regional radioactivity concentration $(\mathrm{Bq} / \mathrm{ml}) /($ injected dose $(\mathrm{Bq}) /$ patient weight $(\mathrm{g}))$. The SUVmax, which was defined as the peak SUV in the pixel with the highest count within the ROI, was measured and recorded for the focal areas of uptake.

Statistical analysis. Statistical analyses were performed with SPSS v22.0 for Windows (IBM Japan, Ltd., Tokyo, Japan). Data were analysed using Fisher's exact test or chi-square test and presented as percentages or mean \pm standard deviation, unless otherwise stated. The survival time of patients known to be alive at the time data collection was censored at the date of last follow-up. Kaplan-Meier analysis was used to calculate the overall survival (OS), and a generalized Wilcoxon test with the level of statistical significance set at 5\% (two-tailed) was used to compare the Kaplan-Meier curves between groups.

\section{Results}

Comparison of OS between PET-positive patients and PETnegative patients. There was no significant difference in the OS (median OS: 811 days for the PET-positive group, 1383
Table I. Analysis of cytology-negative (Class II and III) patients.

\begin{tabular}{lc}
\hline Characteristics (n=9) & \\
\hline Gender & \\
Male:Female & $9: 0$ \\
Age (median) & $69.0(56-78)$ \\
Cytology & $3(33.3 \%)$ \\
Class II & $6(66.7 \%)$ \\
Class III & \\
Histological subtype & $7(77.8 \%)$ \\
Epithelial & $2(22.2 \%)$ \\
Biphasic & \\
Treatment & $1(11.1 \%)$ \\
Trimodality (EPP) & None \\
Bimodality (P/D) & $7(77.8 \%)$ \\
Chemotherapy only & $1(11.1 \%)$ \\
BSC &
\end{tabular}

EPP: Extrapleural pneumonectomy; P/D: pleurectomy/decortication; BSC: best supportive care. Gender, cytology, histological subtype, and treatment received are shown for the cytology-negative group.

days for the PET-negative group; $p=0.2356$ ) but the PETnegative group showed a trend towards longer survival (Figure 1A).

In the T-classification, $22(55.0 \%)$ and $18(45.0 \%)$ cases were T1a and T1b, respectively (Figure 1B). There was no significant difference in survival between $\mathrm{T} 1 \mathrm{a}$ and $\mathrm{T} 1 \mathrm{~b}$ groups $(1,383$ days versus 1,307 days, $p=0.8844)$. The median age was 70 years (range $=51-83$ years), with no significant difference in survival time observed between ages $\geq 70$ years $(1,385$ days) and $\leq 70$ years $(1,805$ days) (Figure $1 \mathrm{C}, p=0.6368)$.

Impact of histological type and pleural effusion on survival. Thirty-seven cases (92.5\%) were of the epithelioid type and three $(7.5 \%)$ were of the biphasic type; sarcomatoid MPM was not observed in any of the early-stage cases of this study (Figure 2). Comparison of OS between the epithelioid and biphasic groups showed a significantly worse prognosis for the biphasic type (median OS: biphasic type, 385 days; epithelioid type, 1,383 days, $p<0.001$ ).

Accumulation of pleural effusion was observed in all cases. Figure 3 shows the pleural effusion cytology with no class I cases, three class II cases (7.5\%), six class III cases $(15.0 \%)$, seven class IV cases $(17.5 \%)$, and 24 class V cases $(60.0 \%)$. Comparison of OS between a cytology-negative group (Class II/III) and cytology-positive group (Class IV/V) showed significantly shorter OS for the cytology-negative group (596 days) when compared with the cytology-positive group (1805 days) $(p<0.05)$. Table I shows the patient characteristics of the nine cases in the cytology-negative group, based on the results from pleural effusion cytology analysis. The present study 


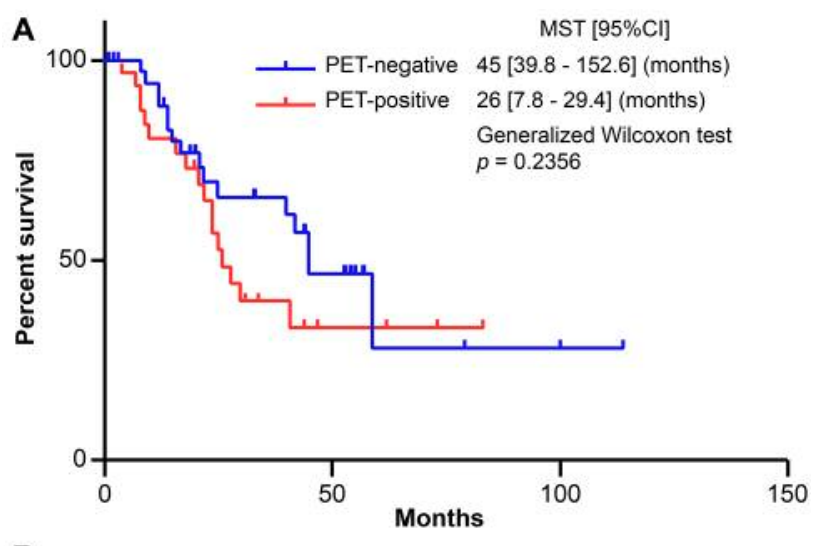

B

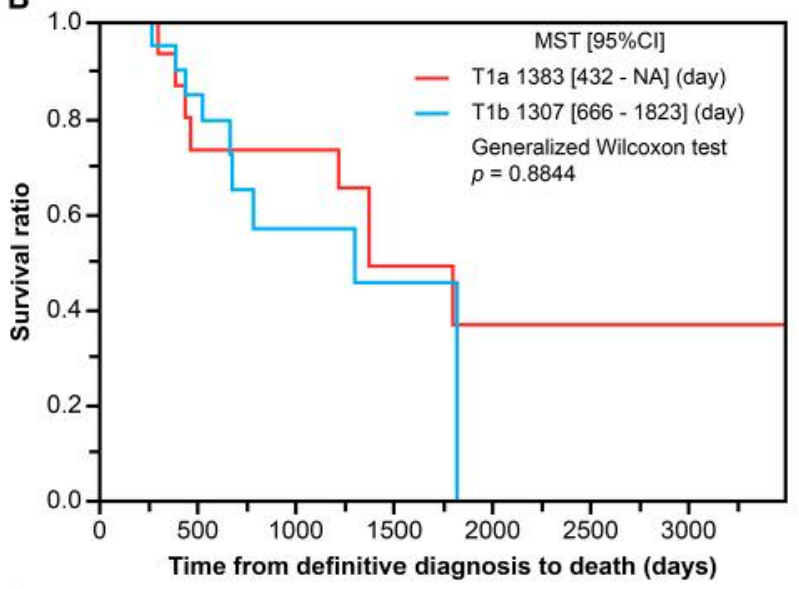

C

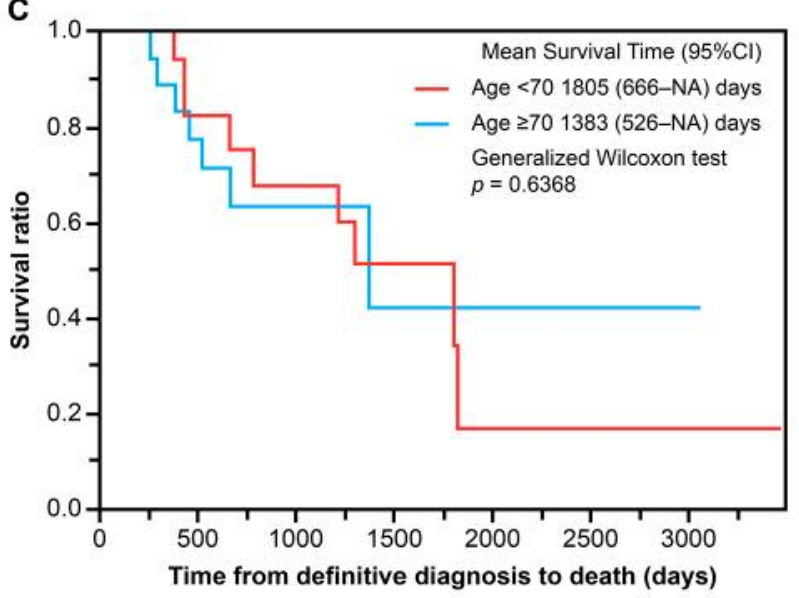

Figure 1. Characterization of study patients. (A) Kaplan-Meier survival curves of PET-negative and PET-positive patients showed no significant difference in survival outcomes. (B) Kaplan-Meier survival curves of TNM classification of patients showed no difference between T1a and T1b classes. (C) Kaplan-Meier survival curve of patients aged $<70$ and $>70$ years showed no difference in survival dependent on age.

demonstrated that the biphasic histological type was associated with poorer prognosis over epithelioid types, even in earlystage mesothelioma cases. In fact, of the 40 cases included in

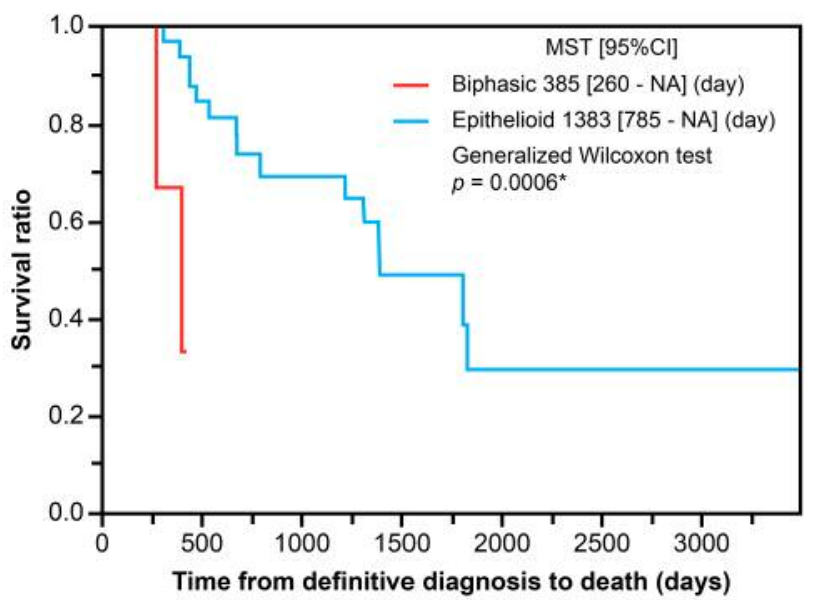

Figure 2. Survival curves of subtypes. Kaplan-Meier survival curves of biphasic and epithelioid subtypes showed that patients of the epithelioid subtype have greater survival than biphasic subtype $(p<0.001)$.

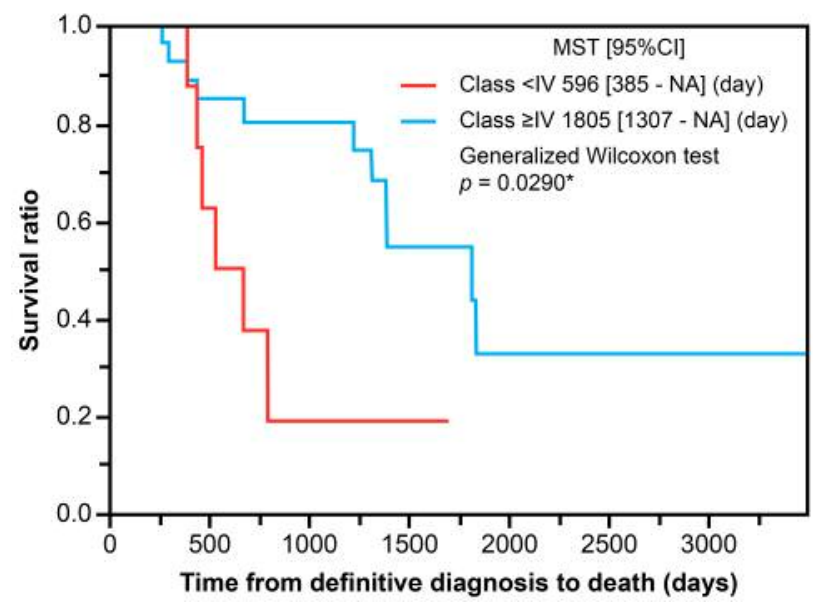

Figure 3. Cytology analysis of survival outcomes. Kaplan-Meier survival analysis showed $\geq$ Class IV cytology-positive patients have improved survival compared to patients with $<$ Class IV cytology $(p<0.05)$.

the analysis, two of the three biphasic cases present were in the cytology-negative group. Investigation of treatment for the cytology-negative group showed eight cases (88.9\%) in the non-surgery group.

Impact of bimodal and trimodal therapy on survival. Patients were grouped as follows: Trimodality group [NeoAdjuvant Chemotherapy (NAC) followed by EPP, followed by Hemithoracic-Radiation therapy (H-RT)]; Bimodality group [NAC followed by Pleurectomy/Decortication (P/D)]; Chemotherapy-alone group; and best supportive care (BSC) 


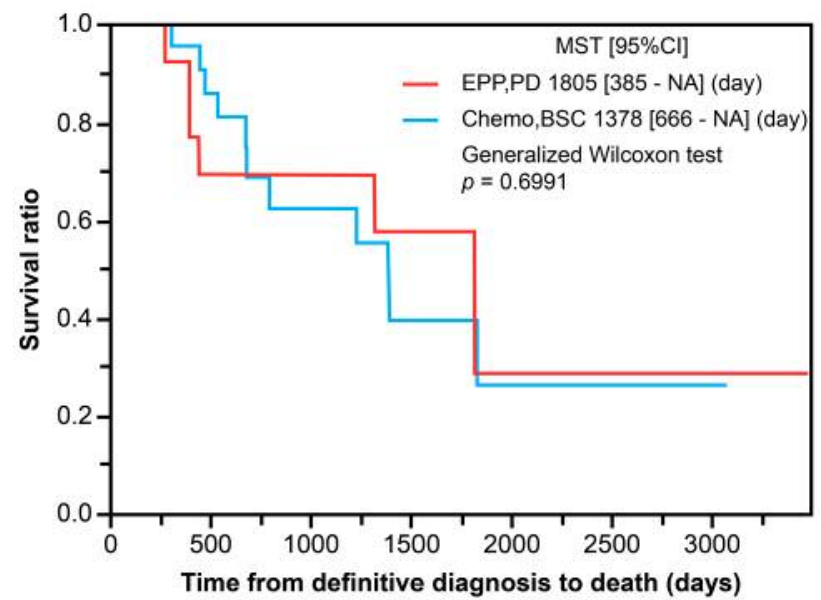

Figure 4. Survival difference between patients who received $E P P$ and $P D$ and those who received chemotherapy and BSC. Kaplan-Meier analysis of patient groups that received either EPP and PD or chemotherapy and best supportive care (BSC) showed no difference in survival outcomes. EPP: Extrapleural pneumonectomy; P/D: pleurectomy or decortication.

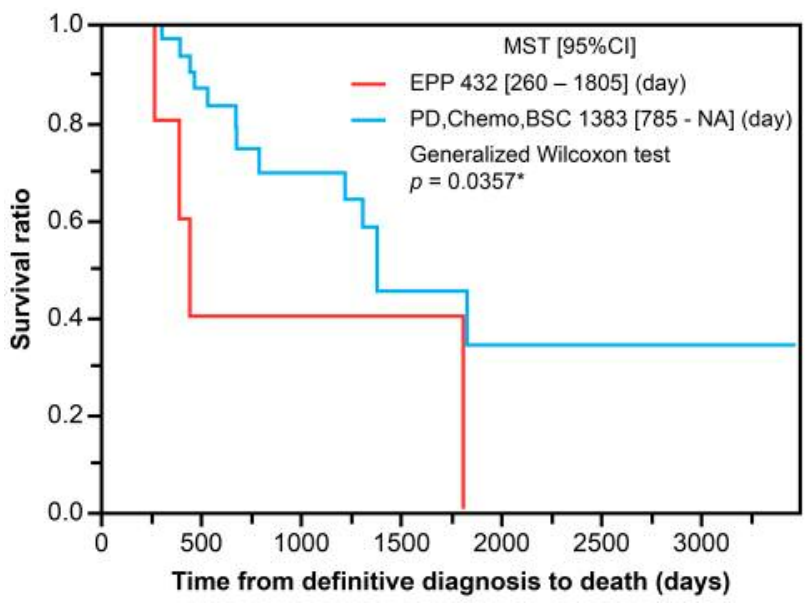

Figure 5. PD, chemotherapy, and BSC show better survival outcomes. Patients who received trimodal therapy (EPP and radiation) had worse survival outcomes over patients who received bimodal therapy ( $P D$, chemotherapy and best supportive care $)(p<0.05)$. group. Median OS was 432 days for the trimodality group (five cases, $12.5 \%$ ), not available for the bimodality group (eight cases, 20\%), 1218 days for the chemotherapy-alone group (20 cases, $55 \%$ ), and 1823 days for the BSC group (five cases, $12.5 \%$ ). Comparison of OS between the surgery group, including those who underwent $\mathrm{EPP}$ or $\mathrm{PD}$, and the nonsurgery group did not show a statistically significant difference (Figure $4 ; p=0.6991)$. However, the median survival time of the non-EPP group was 1383 days longer than that of the EPP group (432 days) $(p<0.05)$ (Figure 5).

\section{Discussion}

There is currently no established evidence that early detection makes it possible to cure MPM or extend patient survival time (15). However, previous reports have shown early-stage MPM patients have better survival outcomes (16-18). There are major challenges in treating MPM, which has an extremely poor prognosis, with a median survival time from diagnosis to death of only 7.9 months (19). This suggests that a practical method for improving survival times could be earlier diagnosis of MPM followed by therapeutic intervention (20).

The present study proposed criteria to diagnose the clinical signs of early-stage MPM, with the purpose of exploring the feasibility of doing so. The first stage of this study involved comparing the PET-positive group and the PET-negative group. No significant differences were found between the groups. Despite the absence of significant differences, the PET-negative group tended to have a longer survival than the PET-positive group. Therefore, in this study, PET-negative cases (SUV<2.5) were defined as early-stage MPM cases. The absence of significant differences in survival rates between the two groups could be attributed to the post-diagnosis treatment. Moreover, a follow-up of cases without treatment would be ethically unacceptable. Therefore, the fact that the PETnegative group tended to have a longer survival was adequate to justify its use as early-stage cases. The results of the present study demonstrated the clinical signs of early-stage MPM; it was found that, even among those in the early-stage MPM, the biphasic type, those having undergone EPP, and having negative cytology were identified as factors associated with poor prognosis. Meanwhile, no significant difference was observed for the T-factor of the TNM. Based on the recent Union for International Cancer Control American Joint Committee on Cancer staging criteria (21), T1a and T1b stages have been merged together. Consistent with the new staging criteria, we did not observe any significant difference in OS rates from patients with $\mathrm{T} 1 \mathrm{a}$ and $\mathrm{T} 1 \mathrm{~b}(21)$.

As with previous reports (22-24), our results demonstrate biphasic histotype as a poor prognostic factor. The outcomes of cases that underwent EPP in our study were marked by a median OS time of 14.4 months and a 3 -year postoperative survival rate of $60 \%$. Considering that the MST after TMT including EPP remains $<20$ months in the majority of largescale studies $(11,12)$, the outcomes in our study were similar to those in previous reports. Thus, the significantly longer median survival time of the non-EPP group (46.1 months) than the EPP group (14.4 months) suggests that improved survival outcomes were achieved by patients undergoing P/D rather than EPP. 
Although negative cytology was identified as a poor prognostic factor, the histotype of two of the nine cases in the cytology-negative group was biphasic, which was also identified as a poor prognostic factor. Investigation of the treatment content for the cytology-negative group showed that eight cases $(88.9 \%)$ were in the non-surgery group, and the median survival times may also be reflective of the differences in treatment modality.

The MARS study, a randomized controlled study to directly compare EPP versus no-EPP, concluded that EPP is detrimental and does not contribute to improving the survival rate (25). However, this study was designed to verify whether randomized allocation is feasible, not to be used to select optimal treatment choices. Opinions differ on the content and interpretation of the study, and no clear conclusion was reached.

The IMIG statement (26), currently the most recent guideline, affirms that surgical treatment plays an important role in multimodal therapy for mesothelioma. It also states that the choice between EPP and P/D surgery should be judged individually, based on patient background and clinical conditions. The combined used of cytoreductive surgery and hyperthermic intraperitoneal chemotherapy has been reported to be effective, with an MST of 63.2 months, 5-year survival rate of $52.4 \%$, and 10 -year survival rate of $44.6 \%$ (27). However, the purpose of this operation is cytoreduction and not macroscopic complete resection. Thus, even with the same treatment modalities for another mesothelioma, the primary purpose is very different.

MPM exhibits diverse clinical symptoms and a wide range of survival times in the natural course of the disease: patients may die within several months of diagnosis or survive for years (28). In this study, some cases achieved long-term survival in their natural course with chemotherapy alone, suggesting that a very careful selection of treatments is required for early-stage MPM. Furthermore, the survival benefits of $\mathrm{P} / \mathrm{D}$ were superior to EPP.

\section{Conflicts of Interest}

The Authors have no conflict of interest to declare regarding the contents of this article.

\section{Acknowledgements}

Editorial support in the form of medical writing was provided by Editage, Cactus Communications.

\section{References}

1 Sawabata N, Miyaoka E, Asamura H, Nakanishi Y, Eguchi K, Mori M, Nomori H, Fujii Y, Okumura M, Yokoi K and Japanese Joint Committee for Lung Cancer Registration: Japanese lung cancer registry study of 11,663 surgical cases in 2004: demographic and prognosis changes over decade. J Thorac Oncol 6: 1229-1235, 2011.
2 Sugarbaker DJ, Flores RM, Jaklitsch MT, Richards WG, Strauss GM, Corson JM, DeCamp MM Jr., Swanson SJ, Bueno R, Lukanich JM, Baldini EH and Mentzer SJ: Resection margins, extrapleural nodal status, and cell type determine postoperative long-term survival in trimodality therapy of malignant pleural mesothelioma: results in 183 patients. J Thorac Cardiovasc Surg 117: 54-63, 1999.

3 Weder W, Kestenholz P, Taverna C, Bodis S, Lardinois D, Jerman M and Stahel RA: Neoadjuvant chemotherapy followed by extrapleural pneumonectomy in malignant pleural mesothelioma. J Clin Oncol 22: 3451-3457, 2004.

4 Vogelzang NJ, Rusthoven JJ, Symanowski J, Denham C, Kaukel E, Ruffie P, Gatzemeier U, Boyer M, Emri S, Manegold C, Niyikiza $\mathrm{C}$ and Paoletti P: Phase III study of pemetrexed in combination with cisplatin versus cisplatin alone in patients with malignant pleural mesothelioma. J Clin Oncol 21: 2636-2644, 2003.

5 Rusch VW: Pemetrexed and cisplatin for malignant pleural mesothelioma: a new standard of care? J Clin Oncol 21: 26292630, 2003.

6 Bölükbas S, Manegold C, Eberlein M, Bergmann T, FisselerEckhoff A and Schirren J: Survival after trimodality therapy for malignant pleural mesothelioma: Radical Pleurectomy, chemotherapy with Cisplatin/Pemetrexed and radiotherapy. Lung Cancer 71: 75-81, 2011.

7 Rusch VW, Rosenzweig K, Venkatraman E, Leon L, Raben A, Harrison L, Bains MS, Downey RJ and Ginsberg RJ: A phase II trial of surgical resection and adjuvant high-dose hemithoracic radiation for malignant pleural mesothelioma. J Thorac Cardiovasc Surg 122: 788-795, 2001.

8 Rice DC, Stevens CW, Correa AM, Vaporciyan AA, Tsao A, Forster KM, Walsh GL, Swisher SG, Hofstetter WL, Mehran RJ, Roth JA, Liao Z and Smythe WR: Outcomes after extrapleural pneumonectomy and intensity-modulated radiation therapy for malignant pleural mesothelioma. Ann Thorac Surg 84: 16851692, 2007.

9 Chi A, Liao Z, Nguyen NP, Howe C, Gomez D, Jang SY and Komaki R: Intensity-modulated radiotherapy after extrapleural pneumonectomy in the combined-modality treatment of malignant pleural mesothelioma. J Thorac Oncol 6: 1132-1141, 2011.

10 Weder W, Stahel RA, Bernhard J, Bodis S, Vogt P, Ballabeni P, Lardinois D, Betticher D, Schmid R, Stupp R, Ris HB, Jermann $\mathrm{M}$, Mingrone W, Roth AD, Spiliopoulos A and Swiss Group for Clinical Cancer Research: Multicenter trial of neo-adjuvant chemotherapy followed by extrapleural pneumonectomy in malignant pleural mesothelioma. Ann Oncol 18: 1196-1202, 2007.

11 Van Schil PE, Baas P, Gaafar R, Maat AP, Van de Pol M, Hasan B, Klomp HM, Abdelrahman AM, Welch J, van Meerbeeck JP and European Organisation for Research and Treatment of Cancer (EORTC) Lung Cancer Group: Trimodality therapy for malignant pleural mesothelioma: results from an EORTC phase II multicentre trial. Eur Respir J 36: 1362-1369, 2010.

12 de Perrot M, Feld R, Cho BC, Bezjak A, Anraku M, Burkes R, Roberts H, Tsao MS, Leighl N, Keshavjee S and Johnston MR: Trimodality therapy with induction chemotherapy followed by extrapleural pneumonectomy and adjuvant high-dose hemithoracic radiation for malignant pleural mesothelioma. J Clin Oncol 27: 1413-1418, 2009. 
13 Trousse DS, Avaro JP, D'Journo XB, Doddoli C, Astoul P, Giudicelli R, Fuentes PA and Thomas PA: Is malignant pleural mesothelioma a surgical disease? A review of 83 consecutive extra-pleural pneumonectomies. Eur J Cardiothorac Surg 36: 759-763, 2009.

14 Kitajima K, Fukushima K, Miyoshi Y, Nishimukai A, Hirota S, Igarashi Y, Katsuura T, Maruyama $\mathrm{K}$ and Hirota S: Association between $\square$ F-FDG uptake and molecular subtype of breast cancer. Eur J Nucl Med Mol Imaging 42: 1371-1317, 2015.

15 Scherpereel A, Astoul P, Baas P, Berghmans T, Clayson H, de Vuyst P, Dienemann H, Galateau-Salle F, Hennequin C, Hillerdal G, Le Péchoux C, Mutti L, Pairon JC, Stahel R, van Houtte P, van Meerbeeck J, Waller D, Weder W and European Respiratory Society/European Society of Thoracic Surgeons Task Force: Guidelines of the European Respiratory Society and the European Society of Thoracic Surgeons for the management of malignant pleural mesothelioma. Eur Respir J 35: 479-495, 2010.

16 Boutin C and Rey F: Thoracoscopy in pleural malignant mesothelioma: a prospective study of 188 consecutive patients. Part 1: Diagnosis. Cancer 72: 389-393, 1993.

17 Boutin C, Rey F, Gouvernet J, Viallat JR, Astoul P and Ledoray $\mathrm{V}$ : Thoracoscopy in pleural malignant mesothelioma: a prospective study of 188 consecutive patients. Part 2: Prognosis and staging. Cancer 72: 394-404, 1993.

18 O'Brien ME, Watkins D, Ryan C, Priest K, Corbishley C, Norton A, Ashley S, Rowell N and Sayer R: A randomised trial in malignant mesothelioma (M) of early (E) versus delayed (D) chemotherapy in symptomatically stable patients: the MED trial. Ann Oncol 17: 270-275, 2006.

19 Gemba K, Fujimoto N, Kato K, Aoe K, Takeshima Y, Inai K and Kishimoto T: National survey of malignant mesothelioma and asbestos exposure in Japan. Cancer Sci 103: 483-490, 2012.

20 Pass HI and Carbone M: Current status of screening for malignant pleural mesothelioma. Semin Thorac Cardiovasc Surg 21: 97-104, 2009.

21 Rice D, Chansky K, Nowak A, Pass H, Kindler H, Shemanski L, Opitz I, Call S, Hasegawa S, Kernstine K, Atinkaya C, Rea F, Nafteux P and Rusch VW, Mesothelioma Domain of the IASLC Staging and Prognostic Factors Committee, advisory boards and participating institutions: The IASLC Mesothelioma Staging Project: Proposals for Revisions of the N Descriptors in the Forthcoming Eighth Edition of the TNM Classification for Pleural Mesothelioma. J Thorac Oncol 11: 2100-2111, 2016.
22 Edwards JG, Abrams KR, Leverment JN, Spyt TJ, Waller DA and O'Byrne KJ: Prognostic factors for malignant mesothelioma in 142 patients: validation of CALGB and EORTC prognostic scoring systems. Thorax 55: 731-735, 2000.

23 Johansson L and Lindén CJ: Aspects of histopathologic subtype as a prognostic factor in 85 pleural mesotheliomas. Chest 109: 109-114, 1996.

24 Vigneri P, Martorana F, Manzella L and Stella S: Biomarkers and prognostic factors for malignant pleural mesothelioma. Future Oncol 11(24 Suppl): 29-33, 2015.

25 Treasure T, Lang-Lazdunski L, Waller D, Bliss JM, Tan C, Entwisle J, Snee M, O’Brien M, Thomas G, Senan S, O’Byrne K, Kilburn LS, Spicer J, Landau D, Edwards J, Coombes G, Darlison L, Peto J and MARS trialists: Extra-pleural pneumonectomy versus no extra-pleural pneumonectomy for patients with malignant pleural mesothelioma: clinical outcomes of the Mesothelioma and Radical Surgery (MARS) randomised feasibility study. Lancet Oncol 12: 763-772, 2011.

26 Rusch V, Baldini EH, Bueno R, De Perrot M, Flores R, Hasegawa S, Klepetko W, Krug L, Lang-Lazdunski L, Pass H, Weder W, Sugarbaker DJ and participants in 2012 International Mesothelioma Interest Group Congress: The role of surgical cytoreduction in the treatment of malignant pleural mesothelioma: meeting summary of the International Mesothelioma Interest Group Congress, September 11-14, 2012, Boston, Mass. J Thorac Cardiovasc Surg 145: 909-910, 2013.

27 Baratti D, Kusamura S, Cabras AD, Bertulli R, Hutanu I and Deraco M: Diffuse malignant peritoneal mesothelioma: longterm survival with complete cytoreductive surgery followed by hyperthermic intraperitoneal chemotherapy (HIPEC). Eur J Cancer 49: 3140-3148, 2013.

28 Bibby AC, Tsim S, Kanellakis N, Ball H, Talbot DC, Blyth KG, Maskell NA and Psallidas I: Malignant pleural mesothelioma: an update on investigation, diagnosis and treatment. Eur Respir Rev 25: 472-486, 2016. 